\title{
VALD: the meeting point of data producers and data users
}

\author{
Tatiana Ryabchikova ${ }^{1 *}$, Yury Pakhomov ${ }^{1}$ and Nikolai Piskunov ${ }^{2}$ \\ 1 Institute of Astronomy, Russian Academy of Sciences, Moscow, Russia; ryabchik@inasan.ru, \\ pakhomov@inasan.ru \\ 2 Department of Physics and Astronomy, Division of Astronomy and Space Physics, Uppsala University, \\ Uppsala, Sweden; nikolai.piskunov@physics.uu.se \\ * Correspondence: ryabchik@inasan.ru; Tel.: +7-495-951-3980
}

Version June 27, 2018 submitted to

\begin{abstract}
Vienna Atomic Line Database (VALD) contains data on atomic and molecular energy levels and parameters of spectral lines required for stellar spectra analysis. Hundreds of millions lines for fine spectral synthesis and for opacity calculations are collected in present version of VALD (VALD3). Critical evaluation of the data and the diversity of extraction tools support high popularity of VALD among users. The data model of VALD3 incorporates obligatory links to the bibliography making our database more attractive as publishing platform for data producers. The VALD data quality and completeness are constantly improving allowing better reproduction of stellar spectra. To illustrate continuous evolution of the data content we present a comparative analysis of the recent experimental and theoretical atomic data for Fe-group elements, which will be included in the next VALD release. This release will also include a possibility for extracting the line data with full isotopic and hyperfine structures.
\end{abstract}

Keywords: stellar spectra; atomic and molecular data; databases.

\section{Introduction}

Spectroscopy is one of the main tools for detecting various species and probing physical conditions in the Universe. Stellar spectroscopy provides the information about basic stellar parameters (temperature, gravity, abundances, velocities) and combined with theoretical models and astrometric data it reveals stellar populations and their evolution. However, for correct interpretation of the observed spectra one needs accurate parameters of many spectral lines, which are imprinted in the emergent radiation. The most prominent and sensitive spectral features are sensitive to stellar parameter and thus we need large amount of reliable data and the means for selecting relevant information for our targets. This huge amount of data is collected in specialised databases (DB) of atomic and molecular line parameters. Vienna Atomic Line Database (VALD) created in 1995 [1] by astrophysists quickly became one of the most popular DB for the analysis of stellar spectra. The second version, VALD2 [2], was running since 1999, and VALD3 [3] started its work since 2015.

Throughout its development VALD became a meeting point of data producers and data users. Figure 1 represents schematically VALD structure. VALD team critically analyses every new atomic and molecular parameter dataset ("line list" in VALD terminology) that we receive from data producers by comparing spectral fits to high-precision spectra of reference stars (the Sun, Sirius, Procyon, etc). At this point we establish the rank for each data type in the new line list that is then included in VALD. In this way we save all previous datasets so that we can reproduce the results from earlier releases. The new data is seamlessly integrated in the extraction delivering the best content according to our evaluation. We regularly check our quality assessments against other centres and groups (e.g. NIST), constantly collect feedback from data users and discuss methodology in forums such as VAMDC (www.vamdc.org). VALD team ranking is compiled in the so-called default VALD configuration. Users get the possibility to create their own copy of configuration where they can adjust the ranking parameters. The requested data is returned to the user by e-mail or ftp. 


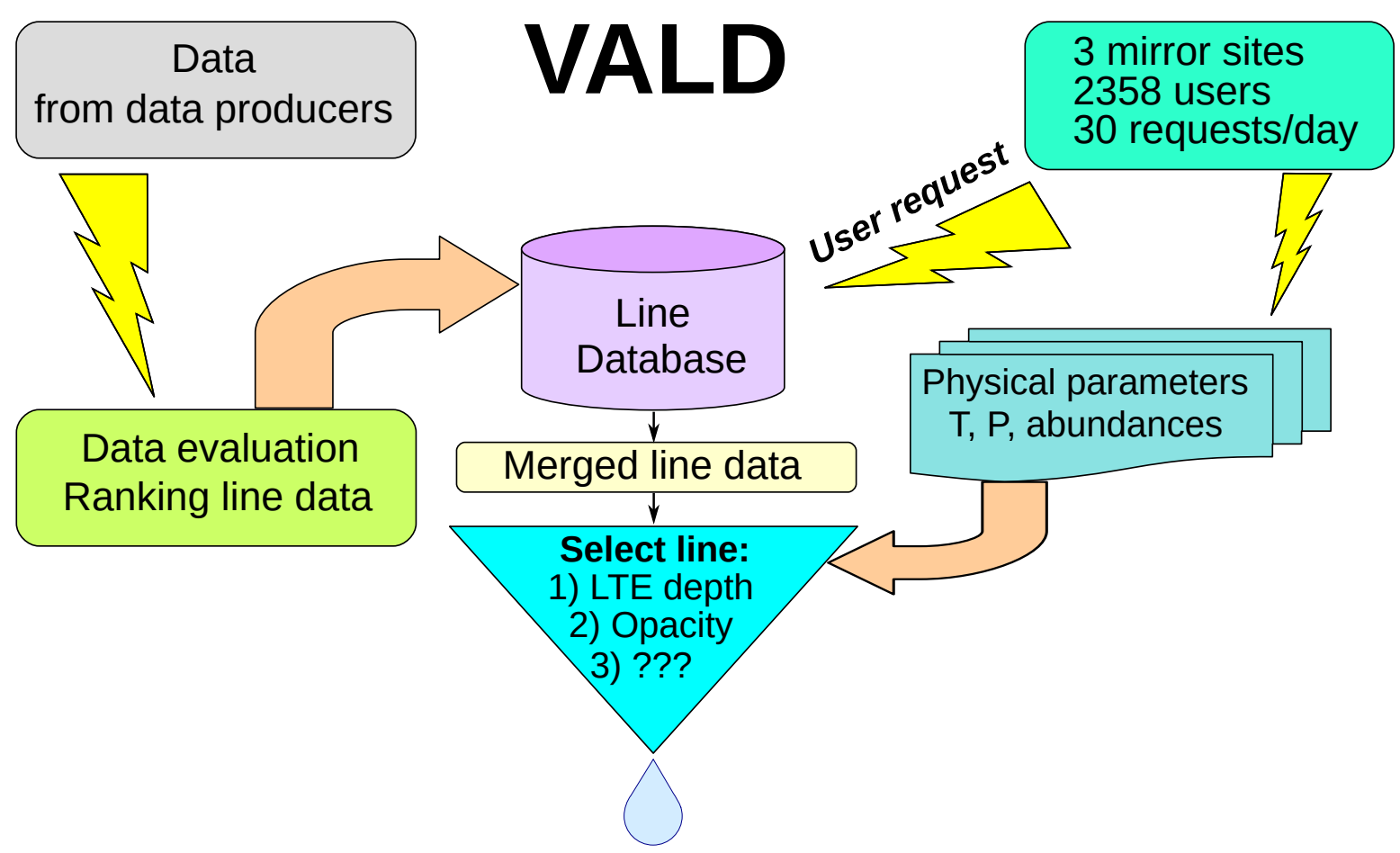

Figure 1. Schematic presentation of VALD database structure.

\section{Data extraction}

The VALD users appreciate the variety of data extraction tools. It is possible to examine the full VALD collection of parameters for individual spectral line and to get their combination to a final output ("Show Line ONLINE"), to extract all available data for a certain chemical species in a chosen spectral range ("Extract Element"), to get data for all species selected spectral range ("Extract All"), and to extract data for spectral lines noticeable in the emergent radiation of a star with a given effective temperature, surface gravity and metallicity ("Extract Stellar"). This last extraction tool is particularly popular among stellar spectroscopists because it allows to get lines with the most significant imprint on the stellar flux. It provides the estimated line strength and selects minimal data set for successful spectral synthesis.

Figure 2 demonstrates the level of completeness of VALD and the efficiency of its extraction tools. We show the comparison of the high-resolution observations with synthetic spectra for stars of different effective temperatures: $\iota$ Her $\left(T_{\text {eff }}=17500 \mathrm{~K}\right), 21$ Peg (10400 K), HD 32115 (7250 K), Sun (5777 K), $\delta$ Eri (5040 K) and M1-dwarf UCAC4 443-054906 (3800 K). Spectra of $\iota$ Her, 21 Peg, HD 32115 were obtained with the Echelle SpectroPolarimetric Device for the Observation of Stars (ESPaDOnS) attached at the 3.6m Canada-France-Hawaii Telescope (CFHT) and were taken from the ESPaDOnS $\operatorname{archive}^{1} . \delta$ Eri and UCAC4 443-054906 were observed with the HARPS spectrograph at the ESO 3.6m telescope at La Silla. These were retrieved from the ESO archive ${ }^{2}$. For the Sun we used the Kitt Peak Solar Atlas [4]. Synthetic calculations were based on the VALD3 data extracted for each star using "Extract Stellar" tool.

http://www.cfht.hawaii.edu/Instruments/Spectroscopy/Espadons /

2 http://archive.eso.org/eso/eso_archive_main.html 


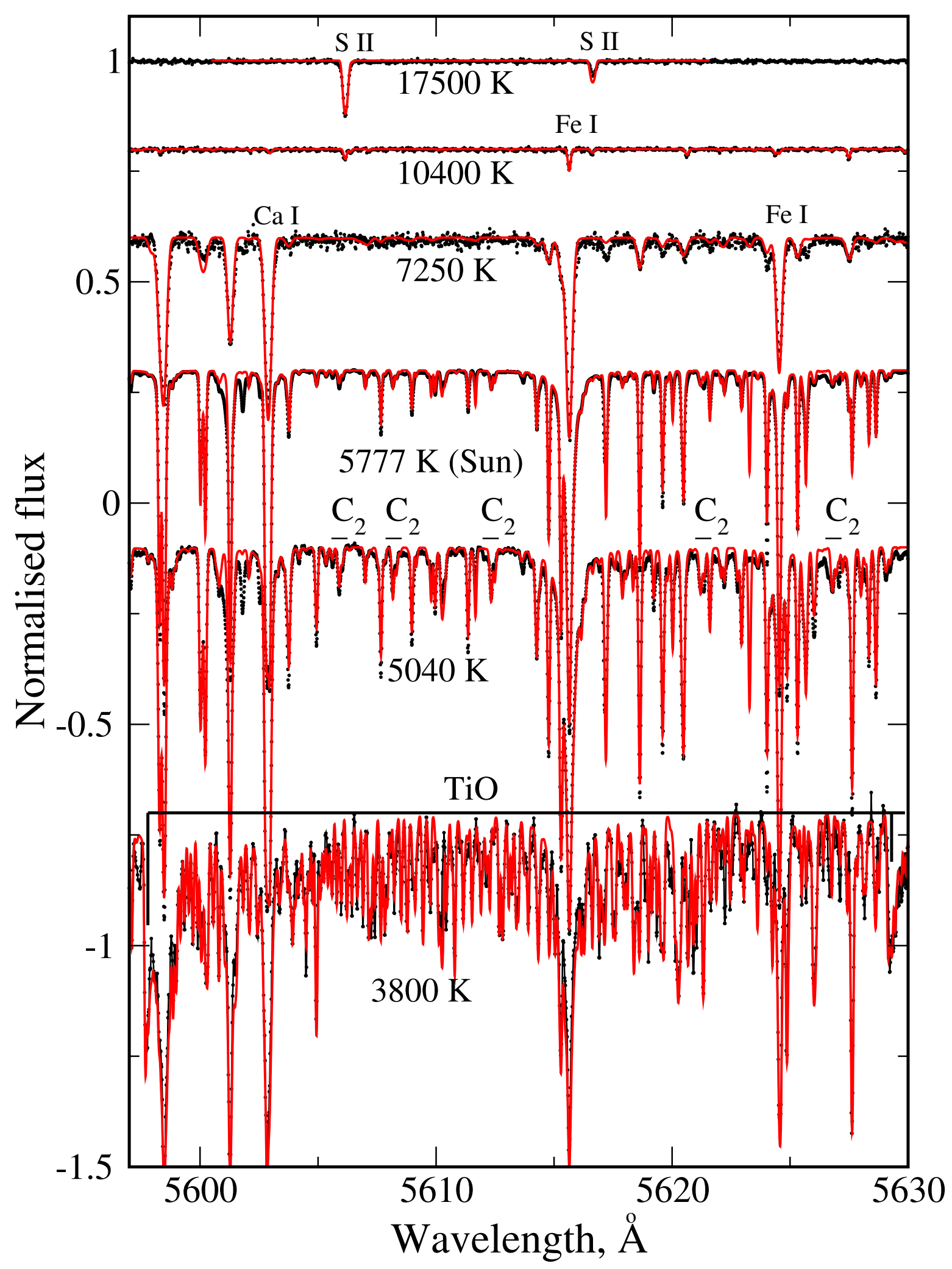

Figure 2. Calculated spectra for different effective temperatures (solid red line) in comparison with the observations (black dots). Atomic parameters were extracted from VALD with the "Extract Stellar" extraction tool. Spectra are shifted in y-axis for better presentation.

57 While in the hottest star, $\iota$ Her, only 8 lines reach central depths over $1 \%$ relative to continuum in 58 the $5590-5630 \AA$ spectral region, 1300 lines are needed to represent the observed spectrum of M1-dwarf. 
Most of the observed features belong to the molecular lines, and the fact that both atomic and molecular line parameters are retrieved through the same interface makes VALD the most efficient 'work horse' for stellar spectroscopic analysis. The only other data collection by R. Kurucz ${ }^{3}$ containing both atomic and molecular line data does not offer "Extract Stellar"-like query option.

Another important new feature in VALD3 is propagation of bibliographic links. Each VALD3 record includes the source reference complemented with optional references to measured wavelength, oscillator strength and isotopic shifts. During the extraction records from different line lists describing the same transition are merged according to the ranking scheme. Thus, the output record may contain entries from several different sources. In VALD3 the bibliographic keys follow the merging procedure so that each field of the output record contains a matching reference. In the final stage of extraction VALD3 collects BibTeX entries matching unique reference keys and delivers them to the user in form of a separate BibTeX file.

\section{Iron analysis}

Iron is one of the most important element in stellar spectroscopy. It is a reference element for metallicity of stars and for chemical evolution of the Galaxy. Therefore the accuracy of iron abundance determination is particularly important. With the present quality of high-resolution spectroscopic observations this task is very sensitive to the accuracy of spectral line parameters. NIST Atomic Spectra Database (ASD) [5] assigns the highest accuracy estimates of 3-5\% for the Oxford laboratory measurements of Fe I transition probabilities [6-10]. Independent laboratory data of the Hannover group [11,12] show small but systematic difference from the Oxford measurements (see Figure 3, left panel). The claimed uncertainties of the Hannover data are $7-15 \%$ and this internal estimate agrees with the one by NIST. For common lines in both sets of measurements NIST recommends the use of Oxford values. The differences between Oxford and Hannover transition probabilities are shown in Figure 3 (left panel). Recently new laboratory data for Fe I transition probabilities were published by spectroscopy groups of Wisconsin University (USA) and Imperial College (UK): Ruffoni et al. [13], Den Hartog et al. [14], and Belmonte et al. [15]. A comparison between the Wisconsin + IC data and Hannover transition probabilities is also shown in Figure 3 (left panel). No systematic difference is visible. We performed Fe abundance measurements in the solar atmosphere with different sets of transition probabilities: Oxford, Hannover and IC [15]. Abundances are displayed in Figure 3 (right panel). We get systematically lower Fe abundance with Hannover and IC transition probabilities compared to that derived with the Oxford data. Careful analysis of all data sets using spectra of other than the Sun reference stars is required to establish a best final list of Fe I experimental transition probabilities recommended for abundance determinations in stellar atmospheres. This work will be done for the next VALD release.

3 http://kurucz.harvard.edu/ 

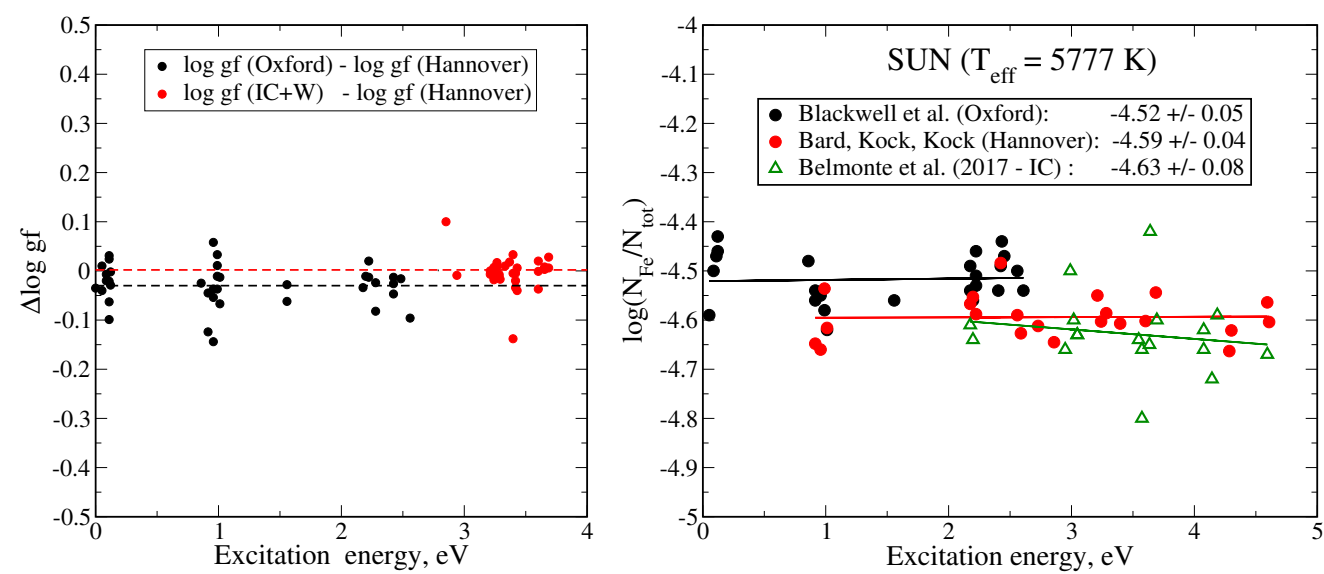

Figure 3. Left panel: Comparison of different sets of the laboratory transition probabilities. Dashed lines show mean values of the differences.

Right panel: Fe abundance in the solar atmosphere derived with the Oxford, Hannover and IC sets of transition probabilities. Linear regressions for each set of data are indicated by solid lines.

Laboratory data are available for a limited number of spectral lines while one needs much more lines for theoretical presentation of the observed spectra. The most part of line parameters come from theoretical calculations. Recently, Peterson \& Kurucz $[16,17]$ performed an extensive analysis of Fe I level structure based on high quality stellar spectra which resulted in newly classified energy levels and new Fe I lines potentially detectable in stellar spectra. At present more than 1100 classified levels are known with ten levels having energies above Fe I ionisation potential. To check the uncertainties of the theoretical data we compared transition probabilities calculated by R. Kurucz ${ }^{4}$ with the experimental measurements by Wisconsin + IC.
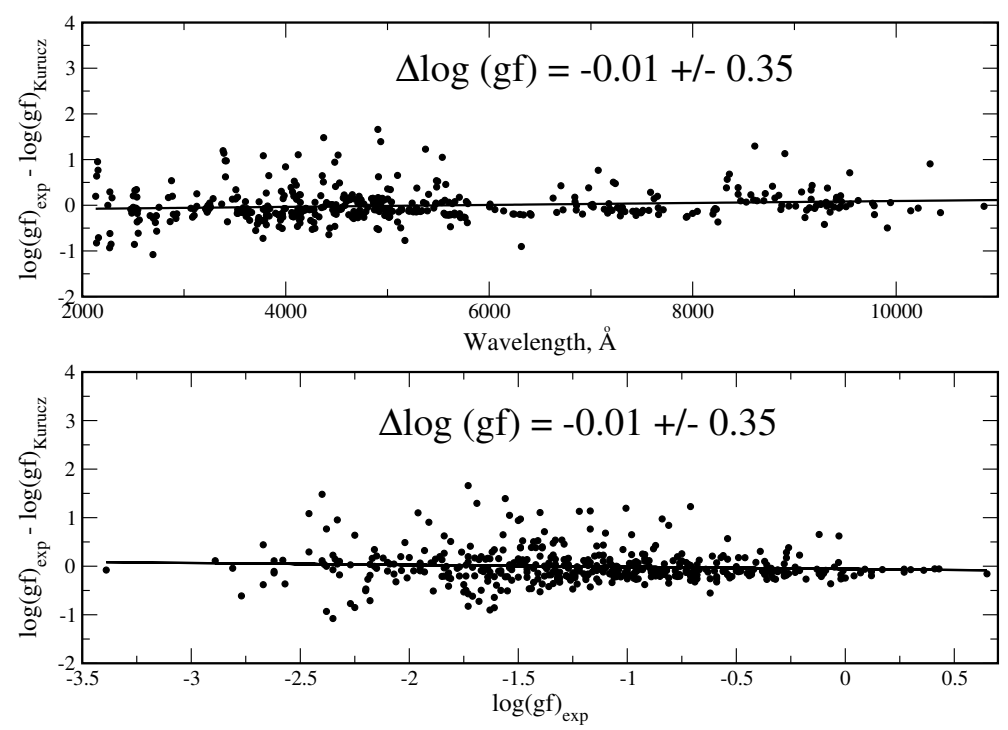

Figure 4. Left panel: Comparison between the experimental transition probabilities of Wisconsin+IC and Kurucz' theoretical calculations as a function of wavelength (top panel) and excitation energy (bottom panel). Linear regressions are indicated by solid lines. 
Figure 4 shows a comparison between experimental ( $\sim 500$ lines) and theoretical transition probabilities. There is no systematic differences. The absolute scales seems to match, although the dispersion of the theoretical data is rather large. The deviation exceeds 2 dex for a few lines. However, laboratory measurements may be erroneous, too, and such comparisons could reveal the errors. In 4 cases the laboratory data were clearly incorrect and had to be replaced by the theoretical calculations. These cases are demonstrated in Figure 5.

All of the examples presented so far illustrated the process of critical data evaluation in VALD.
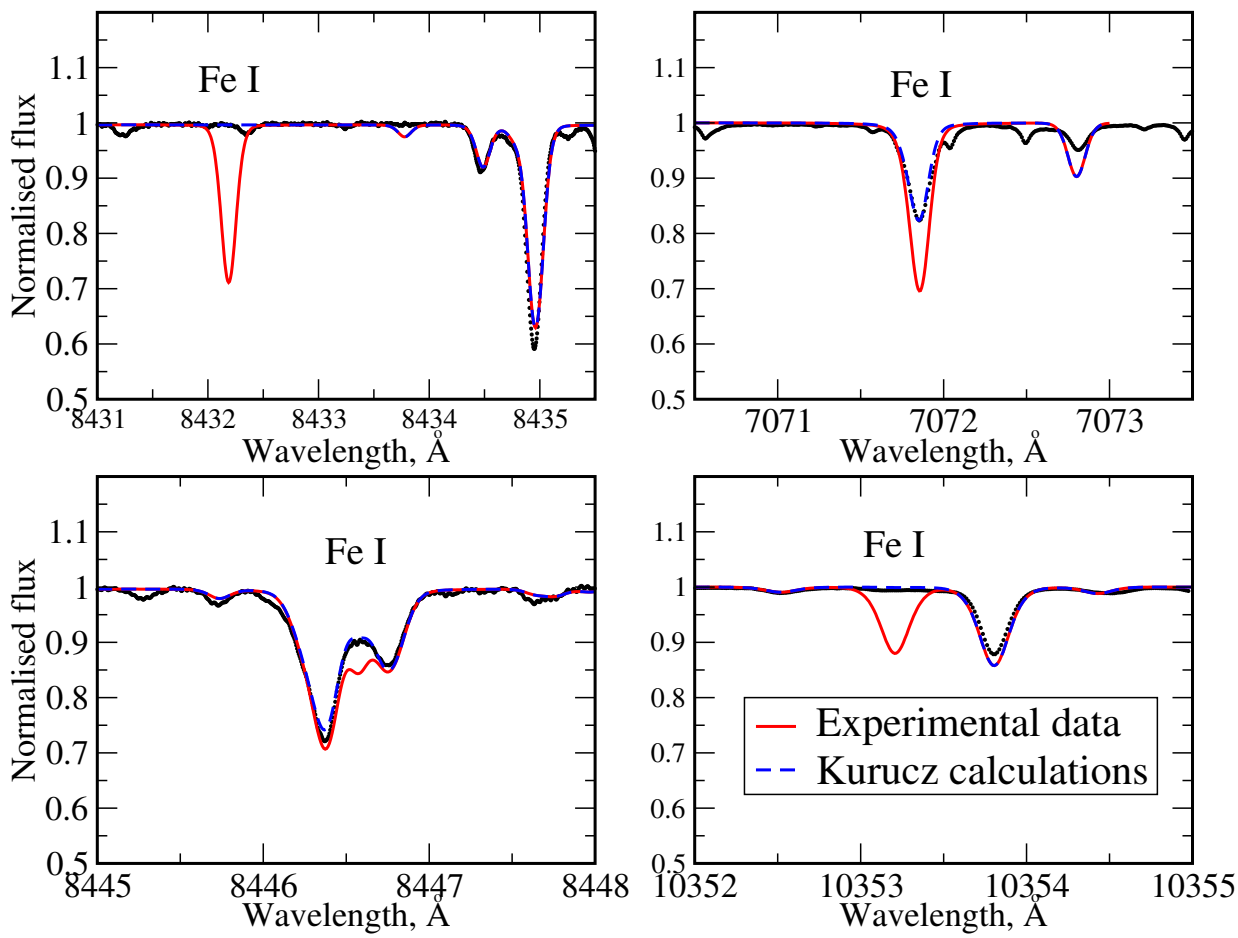

Figure 5. Comparison of the synthetic spectrum calculations made with experimental (red solid line) and theoretical (blue dashed line) transition probabilities to the solar flux observations (black dots).

\section{Hyperfine splitting in VALD}

Transition probability defines spectral line intensity. The lines of an element/isotope with an odd mass number are splitted into components due to multipolar interaction of the nuclear magnetic dipole moment with the magnetic field created by the electron shell. This effect known as hyperfine splitting (hfs) may produce significant change to the line shape and increase line intensity, which has to be taken into account in precision spectral analysis. We created a special SQLite database with data for hfs calculations collected from the literature. These data include species names, energy of levels $E$, total angular moment $J$, magnetic moment $I$, and the hfs constants $A$ and $B$ (see, for more details [18]). Full hfs-pattern on-the-fly calculations will soon be offered as an option for VALD3 "Extract Stellar" query. 


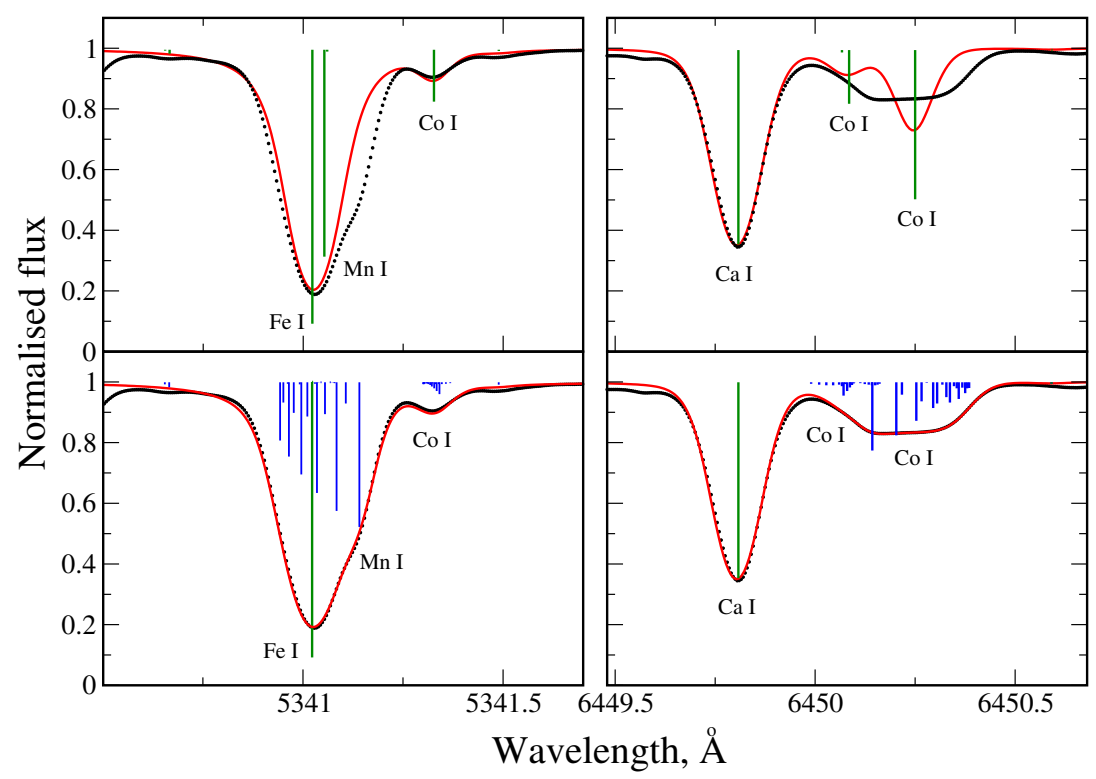

Figure 6. Hyperfine splitting in Mn I and Co I solar lines. Synthetic spectrum calculations without hfs are shown in top panels while the same calculations with hfs taken into account are shown in the bottom panels. The position of the non-splitted lines is indicated by vertical green lines, the hfs-patterns are indicated by vertical blue lines. Observations are shown by black dots.

Figure 6 demonstrates the importance of hfs for accurate fitting of spectral features. Atomic data for synthetic calculations with hfs components were extracted from Moscow VALD3 mirror site ${ }^{5}$ where hfs-extraction is running in a test mode. $A$ and $B$ constants were taken from Dembczyński et al. [19], Başar et al. [20] for Mn I line and from Guthöhrlein and Keller [21], Pickering [22] for Co I lines.

\section{Discussion}

The consistent development of the VALD data content and software reached the point where we can reliably model stellar spectra across all spectral types. The flexible extraction tools tune to user requirements have proven to be very popular. In fact, the VALD3 estimate of the central line depth in "Extract Stellar" mode is frequently used in science publications, e.g. when combining many spectral lines in search for binarity or weak magnetic fields. Massive efforts invested in data consistency verification and standardisation of energy level description as part of the VALD3 transition payed off by reducing the amount of erroneous data and development of the robust merging algorithm for overlapping data. It also opened new possibilities for NLTE calculation etc. The new accurate bibliographic system simplifies the referencing of the original work by data producers making VALD an attractive platform for publishing new data. Large projects working on combination of heterogeneous atomic and molecular data (e.g. VAMDC) look at VALD as model for bibliographic system and data evaluation.

\section{Conclusions}

VALD is an established source of atomic and molecular data for astronomical and particularly stellar spectroscopy. It is the most complete data collection for temperatures encountered in stellar atmospheres and circumstellar medium. Regular updates and critical evaluation of the new data ensures best quality data is delivered to the user. In the same time, flexible referencing system allows to trace the original reference of every data entry in the final selection. New features, such as isotopic 
splitting and hyperfine splitting will soon be available to the users improving the quality of the spectral synthesis. Substantial progress in theoretical atomic and molecular models is bringing the quality of the computed transition probabilities closer to the laboratory measurements further improving the completeness of of our line lists.

Acknowledgments: Current research was supported by the Fundamental Research Program No.28 of Presidium of the RAS.

Author Contributions: T.R. performed Iron analysis and wrote the main text of the paper; Yu.P. provided hfs calculations and made plots; N.P. designed and further developped VALD to its current version, implemented to the text of the paper.

Conflicts of Interest: "The authors declare no conflict of interest."

\section{Abbreviations}

The following abbreviations are used in this manuscript:

NIST National Institute of Standards and Technology

IC Imperial College

VAMDC Virtual Atomic and Molecular Data Centre

\section{References}

1. Piskunov, N.E.; Kupka, F.; Ryabchikova, T.A.; Weiss, W.W.; Jeffery, C.S. VALD: The Vienna Atomic Line Data Base. A\&AS 1995, 112, 525.

2. Kupka, F.; Piskunov, N.; Ryabchikova, T.A.; Stempels, H.C.; Weiss, W.W. VALD-2: Progress of the Vienna Atomic Line Data Base. A\&AS 1999, 138, 119-133.

3. Ryabchikova, T.; Piskunov, N.; Kurucz, R.L.; Stempels, H.C.; Heiter, U.; Pakhomov, Y.; Barklem, P.S. A major upgrade of the VALD database. Phys. Scr 2015, 90, 054005.

4. Kurucz, R.L.; Furenlid, I.; Brault, J.; Testerman, L. Solar flux atlas from 296 to $1300 \mathrm{~nm}$; New Mexico: National Solar Observatory, 1984.

5. Kramida, A.; Ralchenko, Yu.; Reader, J.; and NIST ASD Team. NIST Atomic Spectra Database (ver. 5.5.6), [Online]. Available: https : //physics .nist . gov/asd [2018, June 18]. National Institute of Standards and Technology, Gaithersburg, MD., 2018.

6. Blackwell, D.E.; Ibbetson, P.A.; Petford, A.D.; Shallis, M.J. Precision measurement of relative oscillator strengths. IV - Attainment of 0.5 per cent accuracy: Fe I transitions from levels a5 D/0-4/ / 0.00-0.12 eV/. MNRAS 1979, 186, 633-650.

7. Blackwell, D.E.; Petford, A.D.; Shallis, M.J. Precision measurement of relative oscillator strengths. VI Measures of Fe I transitions from levels a5 F/1-5/ / 0.86-1.01 eV/ with an accuracy of 0.5 per cent. MNRAS 1979, 186, 657-668.

8. Blackwell, D.E.; Petford, A.D.; Shallis, M.J.; Simmons, G.J. Precision Measurement of Relative Oscillator Strengths - Part Eight - Measures of FEI Transitions from Levels A3F2-4/1.49-1.61EV with an Accuracy of 1\%. MNRAS 1980, 191, 445.

9. Blackwell, D.E.; Petford, A.D.; Shallis, M.J.; Simmons, G.J. Precision measurement of relative oscillator strengths. IX - Measures of Fe I transitions from levels a5 P1-3 /2.18-2.28 eV/, a3 P2 /2.28 eV/, a3 P0,1 /2.49-2.42 eV/, z7 D1-5 0 /2.48-2.40 eV/ and a3 H4-6 /2.45-2.40 eV/. MNRAS 1982, 199, 43-51.

10. Blackwell, D.E.; Booth, A.J.; Haddock, D.J;; Petford, A.D.; Leggett, S.K. Measurement of the oscillator strengths of very weak $1 \mathrm{eV}$ Fe I lines. MNRAS 1986, 220, 549-553.

11. Bard, A.; Kock, A.; Kock, M. Fe I oscillator strengths of lines of astrophysical interest. Astron. and Astrophys. 1991, 248, 315-322. (BKK).

12. Bard, A.; Kock, M. Fe I oscillator strengths for lines with excitation energies between 3 and $7 \mathrm{eV}$. Astron. and Astrophys. 1994, 282, 1014-1020. (BK).

13. Ruffoni, M.P.; Den Hartog, E.A.; Lawler, J.E.; Brewer, N.R.; Lind, K.; Nave, G.; Pickering, J.C. Fe I oscillator strengths for the Gaia-ESO survey. MNRAS 2014, 441, 3127-3136, [arXiv:astro-ph.SR/1404.5578].

14. Den Hartog, E.A.; Ruffoni, M.P.; Lawler, J.E.; Pickering, J.C.; Lind, K.; Brewer, N.R. Fe I Oscillator Strengths for Transitions from High-lying Even-parity Levels. ApIS 2014, 215, 23, [arXiv:astro-ph.SR/1409.8142]. 
15. Belmonte, M.T.; Pickering, J.C.; Ruffoni, M.P.; Den Hartog, E.A.; Lawler, J.E.; Guzman, A.; Heiter, U. Fe I Oscillator Strengths for Transitions from High-lying Odd-parity Levels. ApJ 2017, 848, 125, [arXiv:astro-ph.SR/1710.07571].

16. Peterson, R.C.; Kurucz, R.L. New Fe I Level Energies and Line Identifications from Stellar Spectra. ApJS 2015, 216, 1, [arXiv:astro-ph.SR/1406.0933].

17. Peterson, R.C.; Kurucz, R.L.; Ayres, T.R. New Fe I Level Energies and Line Identifications from Stellar Spectra. II. Initial Results from New Ultraviolet Spectra of Metal-poor Stars. ApJS 2017, 229, 23.

18. Pakhomov, Y.; Piskunov, N.; Ryabchikova, T. VALD3: Current Developments. Stars: From Collapse to Collapse; Balega, Y.Y.; Kudryavtsev, D.O.; Romanyuk, I.I.; Yakunin, I.A., Eds., 2017, Vol. 510, Astronomical Society of the Pacific Conference Series, p. 518, [arXiv:astro-ph.IM/1710.10854].

19. Dembczyński, J.; Ertmer, W.; Johann, U.; Penselin, S.; Stinner, P. Laser-Rf double-resonance studies of the hyperfine structure of metastable atomic states of ${ }^{55} \mathrm{Mn}$. Zeitschrift fur Physik A Hadrons and Nuclei 1979, 291, 207-218.

20. Başar, G.; Başar, G.; Acar, G.; Öztürk, I.K.; Kröger, S. Hyperfine Structure Investigations of Mn I. Part I: Experimental and Theoretical Studies of the Hyperfine Structure in the Even Configurations. Physica Scripta 2003, 67, 476.

21. Guthöhrlein, G.H.; Keller, H.P. Doppler-free laserspectroscopic investigations of hyperfine structure in the atomic cobalt spectrum. Zeitschrift fur Physik D Atoms Molecules Clusters 1990, 17, 181-193.

22. Pickering, J.C. Measurements of the Hyperfine Structure of Atomic Energy Levels in CO i. Astrophysical Journal Supplement Series 1996, 107, 811. 